\title{
La dissémination des postes de travail pour une urbanisation répartie
}

\author{
The dissemination of workplaces for distributed urbanization
}

\author{
Serge Le Roux ${ }^{1}$ \\ ${ }^{1}$ Réseau de recherches sur l'innovation, rrislr@yahoo.fr
}

RÉSUMÉ. Dans le dernier quart du XXe siècle, la possibilité de travailler à distance est apparue. Cette nouvelle ouverture technologique a suscité des espérances en matière d'humanisation des conditions de travail et de revitalisation des territoires ruraux. Globalement, ces espérances ont été déçues : peu d'innovations se sont réellement concrétisées. Aujourd'hui, une nouvelle technologie : l'impression en trois dimensions et les Fabrication Laboratories, ouvre un champ inédit d'expériences, permettant, d'une part aux individus de fabriquer eux-mêmes les objets dont ils ont besoin et, d'autre part, aux territoires ruraux de se redynamiser. La question se pose de savoir si ces derniers pourront mettre en place de telles stratégies, sachant qu'ils ne disposent pas de la culture économique et technique appropriée, et qu'ils se heurtent aux entreprises, qui détiennent historiquement le monopole de la production.

ABSTRACT. In the last quarter of the twentieth century, the possibility of remote working arose. This new technological opening has given rise to hopes for the humanization of working conditions and the revitalization of rural areas. Overall, these expectations have been disappointed: few innovations have actually taken place. Today, a new technology: threedimensional printing and the Fabrication Laboratories, opens up a new field of experiments, enabling individuals to make the objects they need themselves and, on the other hand, to revitalize rural areas. The question arises as to whether these rural areas can implement such strategies, knowing that they lack the appropriate economic and technical culture, and that they come up against companies which historically have the monopoly of production.

MOTS-CLÉS. télétravail, conditions de travail, territoires, impression 3D, FabLab.

KEYWORDS. remote working, telecommuting, working conditions, rural areas, 3D printing, FabLab.

L'intellectualisation du travail (travail sur symboles supplantant le travail direct sur la matière tangible) associée à la création de nouveaux outils (ordinateur, liaisons informatiques à grande vitesse, gestion de bases de données, etc.) a permis d'envisager une organisation du travail, différente de celle connue jusqu'alors, propre à la révolution industrielle, centrée sur la machine. Il est désormais possible de mettre en place des dispositifs décentralisés, où la présence constante des opérateurs humains autour de l'objet de travail (système de l'usine) n'est plus nécessaire, les différents postes de travail pouvant être soit regroupés dans le lieu central d'activité (le bureau) soit en être éloignés tout en y restant liés (selon des modalités à concevoir).

Ces modes d'organisation de l'activité productive ont des conséquences directes sur les localisations des résidences des travailleurs: regroupement autour des usines dans le premier cas (soit l'urbanisation); équilibrage des territoires urbains et ruraux dans le second cas.

Toutefois, ces schémas d'évolution ne se sont que peu vérifiés dans les pratiques connues, le modèle urbain demeurant encore nettement hégémonique, dans un univers productif pourtant de plus en plus marqué par le travail de bureau.

\section{Origines}

Lorsque Jack Niles, alors directeur du Programme NTIC du Centre du Futur de l'Université de Californie du Sud (UCLA) utilisa, le premier, le terme de "telecommuting», en 1973, certains observateurs analysèrent cette innovation comme une possible étape nouvelle dans la longue histoire 
de la destruction créatrice. L'époque était effectivement celle de la recherche d'alternatives au modèle d'organisation économique existant.

Ce modèle, apparu il y a plusieurs siècles ${ }^{1}$, s'était transformé en révolution industrielle qui, ellemême, avait connu crises et réformes. Parmi ces réformes, deux sont à signaler plus spécifiquement :

- la réforme fordienne où Henry Ford, célèbre fabricant d'automobiles à Detroit, imagina de relier production et consommation de masse ;

- la réforme keynésienne consistant à introduire des modalités, plus ou moins égalitaires, de redistribution à l'intérieur des espaces géopolitiques des Etats-nations.

La période où s'exprimait Jack Niles était celle où les effets bénéfiques des deux grandes réformes précitées commençaient à atteindre leurs limites : les risques de surproduction (réforme fordienne) étaient supplantés par la demande de produits plus personnalisés et par les commencements de la mondialisation; les capacités redistributives des Etats-nations traditionnels amorçaient leur déclin historique.

Mais surtout, apparaissait une nouvelle manière de produire, dont on pouvait pressentir qu'il s'agissait de l'annonce d'une troisième crise structurelle du système. Le trait principal de cette nouvelle donne tient dans l'insertion des techniques de l'électronique dans la production (le premier ordinateur, de John Von Neumann, pendant la Seconde Guerre mondiale et dans la période suivante ; la cybernétique de Norbert Wiener [WIE 48] ; l'automation de John Diebold [DIE 57] puis dans la gestion (informatique des années 1960-70).

On connaîtra ensuite des évolutions (révolutions) incessantes : décentralisation de l'informatique (microélectronique); fusion avec la communication (internet); pénétrations dans la fabrication (robotique, intelligence artificielle, impression en trois dimensions...).

L'interrogation liminaire de Jack Niles paraît donc légitime dans la mesure où, en plus des transformations des techniques de production (habituelles dans le cadre du système de remplacement homme-machine) se trouvent désormais posées celles du travail, de son contenu, de son statut.

Les espérances issues de l'ouverture ainsi créée pouvaient aller jusqu'à la remise en cause du principe fondateur du système industriel tel qu'il s'est développé depuis la fin du XIX $\mathrm{X}^{\mathrm{e}}$ siècle, à savoir le taylorisme. De telles visées ne correspondaient pas uniquement à l'affirmation d'utopies à prétentions plus ou moins humanistes - très en vogue au XIX ${ }^{\mathfrak{e}}$ siècle et aussi dans les années 19601970 - (quoi que cette question contienne, à l'évidence, une épaisseur effective).

Il s'agissait, plutôt, de mesurer le degré de viabilité d'une nouvelle combinaison productive hommemachine, où la place et le rôle du travail humain seraient qualitativement réévalués. Dans une telle optique, la question du territoire était appelée à prendre une nouvelle actualitée .

Il est sans doute utile de préciser cette notion de telecommuting, avant de tenter d'en analyser les effets, potentiels ou avérés. La définition que l'on peut qualifier d' "officielle » est contenue dans l'article premier de l'Accord national interprofessionnel du 19 juillet 2005 (voir Note $n^{\circ} 6$ ) : « Le

\footnotetext{
${ }^{1}$ L'historien allemand Werner Sombart [SOM 92] date l'inauguration de ce nouveau système à la publication de la Summa... par le moine florentin et mathématicien Luca Pacioli, en 1494 [PAC 94]. Dans cet ouvrage, l'auteur présente l'innovation essentielle de la comptabilité en partie double, où flux et stock (revenus et patrimoine) sont comptés séparément, tout en restant reliés entre eux. Cette innovation est essentielle dans la mesure où elle identifie le principe fondateur du nouveau système, à savoir l'accumulation du capital.

${ }^{2}$ Pour des analyses plus détaillées des origines et de l'histoire du télétravail en France, on peut se reporter à Le Roux, 2012, 2016 et 2017 [LER 12] [LER 16] [LER 17].
} 
télétravail est une forme d'organisation et/ou de réalisation du travail utilisant les technologies de l'information dans le cadre d'un contrat de travail et dans laquelle un travail, qui aurait pu être réalisé dans les locaux de l'employeur, est effectué hors de ces locaux de façon régulière. Cette définition (...) inclut les salariés « nomades », mais le fait de travailler à l'extérieur des locaux de l'entreprise ne suffit pas à conférer à un salarié la qualité de télétravailleur. Le caractère régulier exigé par la définition n'implique pas que le travail doit être réalisé en totalité hors de l'entreprise, et n'exclut donc pas les formes alternant travail dans l'entreprise et travail hors de de l'entreprise ».

Appelé à jouer un rôle dans cette histoire de la destruction créatrice marquant la période de la Révolution industrielle, le télétravail n'a pas atteint ces objectifs : le taylorisme, principe fondateur du système existant a su résister, alors que le territoire restait négligé. Malgré cet échec, certaines avancées créatives ont pu avoir lieu, qui désignent les voies possibles d'innovations d'ampleur. Cellesci seraient fondées, non plus sur l'entreprise, trop imprégnée des critères de valorisation des capitaux, mais sur le territoire où de nouvelles harmonies peuvent se développer comme celles rapprochant vies professionnelle et privée des individus.

\section{Taylorisme, le grand bond en avant du capitalisme}

Le système capitaliste - dont les prémices sont pluriséculaires ( $\mathrm{cf}$. Note $\left.\mathrm{n}^{\circ} 1\right)$ - devait trouver le moyen technique de rendre obsolète l'artisanat. Ce fut fait avec l'invention de la machine à vapeur. Petit à petit, le besoin de réunir des opérateurs autour de la machine apparut et amena à la constitution d'unités de production d'un nouveau style, les manufactures ou fabriques. Ce public laborieux, primoindustriel fut d'abord trouvé parmi les artisans, dont la compétitivité était désormais dépassée.

Ce deuxième âge du capitalisme gardait un pied dans le passé artisanal, et ne parvenait pas à s'en affranchir, faute des méthodes ad hoc d'organisation de l'entreprise. Le grand mérite de l'ingénieur Frederick Winslow Taylor fut d'élaborer le corpus de «l'organisation scientifique du travail» [TAY 90] à la fois théorique et pratique, qui allait permettre le passage de la phase de subsomption formelle du travail à celle de sa subsomption réelle [LER 04]. Un peu comme l'avait fait, 500 ans plus tôt, Luca Pacioli, Taylor rassemblait en une entité unique, l'ensemble des questions du moment où se trouvait le système capitaliste, regroupait les réflexions sur le sujet, et apportait des réponses appropriées, compréhensibles par tous et pouvant être mises en œuvre immédiatement.

Le projet taylorien était celui d'un ingénieur, c'est-à-dire la catégorie sociale (créée à cette époque) qui assurait un pont entre les détenteurs de capitaux et les ouvriers :

- les premiers attendaient une contrepartie, sous forme de profits, pour rémunérer l'avance des capitaux qu'ils avaient réalisée et qui permettait l'engagement de la production ;

- les seconds attendaient leur salaire, prix de l'utilisation de leurs forces de travail par les premiers.

Etabli sur les principes de la logique de l'ingénieur, le projet taylorien mettait au centre de sa construction, intellectuelle et managériale, la machine (premier pan de l'avance en capital). Le travail humain (deuxième pan de cette avance en capital) devait se nicher, selon des calculs savants, dans les interstices laissés vacants dans le dispositif machinique.

Le travail se trouvait ainsi doublement désincarné de sa dimension humaine :

- quand il était intégré dans l'organisation productive, ce n'était que de façon subsidiaire, subordonnée (comme il est considéré dans le rapport salarial); 
- quand les gestes ouvriers pouvaient être suffisamment appréhendés, techniquement et mentalement, par les ingénieurs, ceux-ci étaient en mesure de les transférer dans de nouveaux dispositifs techniques : cf. Le vol du savoir [LUC 89] $]^{3}$.

\section{Le territoire transparent}

Un peu par le simple jeu de sa construction, le système capitaliste accordait une place très réduite au territoire. A part les Beaux quartiers et le marché immobilier, le territoire paraissait assez semblable à la nature: un bien gratuit, éventuellement renouvelable, fournissant des inputs nécessaires à la production... mais qui n'entraient que très marginalement dans le calcul économique. Les stratégies d'implantation des unités de production étaient simplistes: proximité des sources de matières premières et des grandes voies de circulation. Le territoire constituait donc un acteur très secondaire, voire insignifiant.

Mais cette place exigüe à l'intérieur du système n'empêchait pas que le contenu même du territoire soir profondément modifié du fait des pratiques des entreprises industrielles appliquant l'organisation scientifique du travail.

En effet, la logique propre du taylorisme impliquait la division à l'extrême du procès de travail en tâches insécables, en "particules fines ». Lié à une forte croissance de l'activité économique, ce phénomène aboutissait à un appel massif à de la main-d'œuvre supplémentaire. Celle-ci, bien évidemment, ne pouvait être trouvée que dans l'espace rural, d'abord proche, puis dans des zones plus éloignées ou à l'étranger.

Cette rencontre entre l'économique et le territoire aboutit aux deux grands mouvements qui continuent à se développer depuis le $\mathrm{XIX}^{\mathrm{e}}$ siècle :

- la désertification des campagnes ;

- l'urbanisation incessante.

On voit donc bien le lien direct qui associe les stratégies des entreprises de subsumer totalement le travail ouvrier, d'une part et, d'autre part, les désagrégations territoriales. Il pouvait dès lors, paraître légitime de considérer que la venue d'une innovation technologique générique (au sens de Lowe [LOW 76]) comme celle du telecommuting, était de nature à remettre en cause le schéma existant et d'imaginer un nouveau système libérant le travail et équilibrant les territoires.

\section{L'échec du processus de destruction des formes anciennes de production}

Les expériences accumulées depuis 1973 (que ce soit en matière de réduction du taylorisme ou d'affirmation du rôle économique des territoires) ont montré que ces belles espérances relevaient plutôt de l'idéalisme et que les forces tenantes du système refusaient les éventualités alternatives qui se présentaient, sans que de nouveaux acteurs significatifs ne se portent candidats pour initier de nouvelles approches :

- les entreprises : totalement inhibées dans la culture taylorienne, elles n'ont pas su (voulu) s'en dégager. Mieux même, elles ont cherché à introduire ces méthodes, créées pour organiser le travail

\footnotetext{
${ }^{3}$ Les travailleurs, surtout les plus qualifiés, ne restaient pas inertes face à cette stratégie visant à les marginaliser. Par tous les interstices disponibles, ils construisaient, pas à pas, des zones d'autonomie, au point qu'ils pouvaient parvenir à rendre leurs propres méthodes (vs le travail prescrit) indispensables au fonctionnement du système machinique. Ce mouvement est connu sous le terme du « travail réel». Les aptitudes et compétences ainsi constituées peuvent former un socle solide dans le projet d'établir un territoire économique autonome, point qui sera présenté à la fin de cet article.
} 
manuel, dans les nouveaux métiers intellectuels liés à la révolution numérique (hypertaylorisme) ${ }^{4}$ : si ce type de projet pouvait, à la rigueur, relever d'une logique de continuité entre l'ancien, industriel et le nouveau, informatique (de fait, dans les années 1960-1970, les conditions de travail des dactylocodeuses ne se différenciaient guère de celles des ouvrières de l'industrie textile) on peut plus difficilement expliquer le maintien du taylorisme dans le système de l'informatique décentralisée (microordinateur) où l'opérateur, pour la première fois depuis la prise du pouvoir de l'industrie contre l'artisanat, devenait détenteur de ses moyens de travail ${ }^{5}$. Certes, l'on pourra objecter que cet opérateur ne dispose d'aucun accès à la conception et à la fabrication de ses outils de travail, que le contenu et la réalisation de ses tâches sont strictement encadrées dans un schéma dominé par des critères top down relevant quasiment du sacré : le taux de rentabilisation des capitaux avancés... il reste qu'une nouvelle manière de travailler est néanmoins apparue.

- les territoires : eux non plus n'ont pas utilisé les opportunités offertes par la révolution numérique. Ils auraient pu se saisir de celles-ci pour créer une nouvelle forme d'économie politique, fondée sur leurs propres besoins et ceux de leurs habitants, mais deux gros obstacles les en ont empêché :

- l'organisation institutionnelle de la démocratie représentative: les élus locaux gèrent une circonscription administrative, au sein de laquelle se livrent des confrontations politiques. Ce système aboutit à un surdimensionnement de la fonction politique (les fameuses élites sont aujourd'hui critiquées pour cette raison - parmi d'autres) au détriment de la fonction économique. Pour évacuer le risque d'un manque d'intérêt pour la question de l'emploi, les élus ont souvent recours à des actions, plus spectaculaires qu'efficaces, qui se matérialisent, par exemple dans les bien connues «zones industrielles» ou «artisanales»: il s'agit de créer des cubes de béton dans les périphéries, en espérant, forces privations fiscales et subventions souvent d'aubaine à l'appui, attirer des entreprises (logique brick and mortar) ;

- le manque de connaissances économiques des collectivités: la prééminence donnée à l'institutionnel politico-administratif tend à empêcher l'ouverture d'un champ de discussions, de réflexions et de recherches sur la notion de «territoire économique». Or, il semble bien que celle-ci constitue la base d'une véritable politique économique locale. En gros, le schéma peut être présenté en distinguant, dans la population active occupée, trois catégories : (1) ceux qui habitent et travaillent à l'intérieur du territoire juridico-politique ; (2) ceux qui y habitent et qui travaillent dans d'autres territoires; (3) ceux non-résidents qui viennent travailler dans le territoire. On peut imaginer qu'une politique économique locale volontariste pourrait viser l'attraction vers le territoire de non-résidents, travaillant ou non sur le territoire « économique » de la collectivité, c'est-à-dire celui qui comprend toutes les zones accessibles en une seule journée (carte isochrone).

L'Etat, qui n'a reçu que peu d'impulsions de la société civile (entreprises, collectivités, organisations syndicales ${ }^{6} .$. ) n'a envoyé que quelques signaux, plutôt faibles, pour encourager le développement du télétravail en France : appels d'offres de la Datar, introduction de dispositions

\footnotetext{
${ }^{4}$ Logique poussée à son terme, par exemple, par le dirigeant du fonds d'investissement Bridgewater, Ray Dalio, qui souhaite transformer toutes les décisions prises dans l'entreprise en algorithmes [DAL 17].

${ }^{5}$ On a pu qualifier cette innovation de générique, dans la mesure où elle a donné naissance à de nombreuses formes inédites d'activités, par exemple, le Travail collaboratif [LER 09].

${ }^{6}$ L'ensemble des organisations syndicales représentatives des salariés (CGT, CFDT, CGT-FO, CFTC, CFE-CGC) ont signé, avec les organisations patronales (MEDEF, CGPME, UPA) l'Accord national interprofessionnel sur le télétravail le 19 juillet 2005. Au-delà de cette approbation formelle pour cette nouvelle manière de travailler, il faut bien reconnaitre qu'aucune de ces organisations n'a marqué un réel empressement pour la faire entrer dans la réalité.
} 
spécifiques dans le Code du travail, rapport du CGIET [LAR 11] ${ }^{7}$, préparation (en cours) d'un Plan national du déploiement du télétravail (CGET) ${ }^{8}$ quelques tentatives dans la fonction publique...

L'Union européenne a également apporté un soutien sous la forme, par exemple, de rencontres entre acteurs des quinze pays-membres (e-Telework) ${ }^{9}$.

\section{Une créativité faible mais prometteuse}

Si l'on reste dans une approche en termes de destruction créatrice, on voit que le volet « Destructions » n'est pas très convaincant. Celui des créations ne l'est guère plus, mais il permet peutêtre d'imaginer des pistes d'évolution intéressantes (optimisme de la volonté). D'un côté, les (rares) expériences tentées se sont toutes avérées positives; d'un autre côté, les effets bénéfiques sur les travailleurs sont indéniablement plus élevés, que ce soit en nombre et en intensité, que les inconvénients qui peuvent être repérés :

- Renault, un cas d'école: lorsque Carlos Ghosn analyse les comptes de l'entreprise après sa nomination comme PDG, en 2005, et qu'il visite les locaux du siège social à Boulogne-Billancourt, il aurait indiqué son désir de voir ceux-ci réduits de $10000 \mathrm{~m}^{2}$. Les responsables de l'entreprise lancent alors un plan de déploiement du télétravail : l'accueil des collaborateurs à cette proposition est enthousiaste; celui des cadres, beaucoup plus réservé. Quelques années plus tard, plus de 1500 collaborateurs exercent une partie de leur activité depuis leur domicile, avec toujours un taux de satisfaction proche de $100 \%$ (de fait, la proportion de retours à la situation précédente est extrêmement faible et ils sont, le plus souvent, dus à des causes externes) ; l'évolution du point de vue des managers est également significative : ils sont passés d'une attitude franchement hostile au moment du lancement du projet (peurs de perdre le contrôle sur les collaborateurs et de voir les équipes se dissocier) à une «divine » surprise : leurs agents travaillent mieux ${ }^{10}$ et produisent plus, et ce sans qu'on le leur demande !

- Le témoignage d'une télétravailleuse ${ }^{11}$ :

«Quel confort de vie m'apporte d'exercer mon métier en télétravail en tant que mère de deux enfants en primaire et collège et un conjoint professionnellement éloigné du domicile.

Mon envie et mon choix de vivre à la campagne, après de nombreuses années passées en région parisienne, a été conforté par cette possibilité : travailler chez soi. Fini les trajets RER, Métro, embouteillages, gestion des retards, des emplois du temps des enfants, etc.

En optant pour une semaine de travail de $7 \mathrm{~h} /$ jour sur 5 jours soit :

- $8 \mathrm{~h}-12 \mathrm{~h}$ et $13 \mathrm{~h}-16 \mathrm{~h}$ cela me permet de dégager du temps libre surtout en fin de journée pour les activités familiales : goûter, surveillance des devoirs, accompagnement des activités extra scolaires, suivi médical, réunions de parents d'élèves et d'autres comme la possibilité

\footnotetext{
${ }^{7}$ Conseil général de l'Industrie, de l'énergie et des technologies (Lartail et alii, 2011).

${ }^{8}$ Commissariat général à l'égalité des territoires.
}

${ }^{9}$ La dernière (septième) de ces rencontres e-Telework s'est tenue en France, à Bercy ; elle a été organisée par l'Association française
du télétravail et des téléactivités (AFTT) association dont le rédacteur de cet article assure la vice-présidence.

${ }^{10}$ La participation du personnel distancié à l'amélioration de leur travail a été telle qu'elle a surpris la hiérarchie. Il a fallu, d'urgence,
inventer un module de formation spécifique pour pouvoir gérer, au mieux, ce changement du métier du $\mathrm{N}+1$.

${ }^{11}$ Cette télétravailleuse est un agent de la Fonction publique d`Etat, qui a d'abord exercé des fonctions dans des lieux centralisés d'activité, puis, dans une seconde partie de sa carrière a été amené à conjuguer travail de terrain, relations périodiques avec sa hiérarchie et ses collègues, ainsi que travail de bureau réalisé à son domicile. 
d'approvisionnements locaux (circuits courts) enrichissements personnels : sport, bénévolat associatif.

De plus, dans cet aménagement du temps de travail, il est facile de gérer quelques travaux ménagers tels que lessives, accueil facteur ou livraisons ... compensés par les gains de temps engendrés par la suppression des déplacements domicile-travail, des pauses café et de repas beaucoup plus courts. Du temps et moins de stress et donc plus d'efficacité et de production stable.

Des avantages, oui mais... car il y a toujours un revers à la médaille : le télétravail "aliénation ou libération"?

$\mathrm{Du}$ fait de cette disponibilité nouvelle et lorsqu'on vit en couple que devient le partage équitable des tâches du foyer? de l'image de la mère au foyer qui pour les enfants devient la solution à tous leurs problèmes d'intendance ? de la tentation, pour l'employeur, d'augmenter la charge de travail?

Et que deviennent les relations avec les collègues, la hiérarchie, s'il n'y a plus de contacts personnels ? Une journée par semaine de retour sur site paraît indispensable pour ne pas perdre le contact avec ce qui est le poumon de l'entreprise : les collègues n'ayant pas voulu ou pas pu travailler hors du bureau, en raison de l'incompatibilité de leur fonction avec un poste de travail de télétravail, ou par choix personnel ? Les supérieurs hiérarchiques pour faire un point sur le suivi, l'évolution et l'efficacité de ce type d'organisation et son devenir $»$.

La preuve est désormais disponible qu'il est possible travailler en éliminant une partie de l'organisation taylorienne (fondée sur l'émergence de relations de confiance entre l'employeur et ses subordonnés) et que l'on peut voir émerger le chemin d'une réconciliation entre vies professionnelle et personnelle.

\section{D'où peut venir l'impulsion décisive?}

On sent bien que la question du télétravail pourrait être décisive, aussi bien pour les entreprises (création d'une efficacité nouvelle) que pour les territoires (redynamisation et équilibrage) et que la zone de lancement d'un tel processus de déploiement se situe probablement à l'intersection des actions que peuvent mener ces deux entités. On sait aussi que les pratiques de rencontres et cheminements communs entre elles sont ténues, voire inexistantes.

Il est assez peu imaginable qu'une initiative pour transcender ce vide soit l'œuvre des entreprises, engluées dans leurs critères de gestion d'un autre âge.

Il ne reste plus qu'à faire supporter cette responsabilité aux territoires; qui ne sont en rien préparés à cette tâche, bien que certains d'entre eux prennent des décisions qui vont dans le sens du progrès, comme la création de centres de coworking.

Le territoire se définit comme un espace géographique fermé, ses frontières étant généralement constituées par des considérations politico-administratives (une circonscription). Sa gouvernance est, le plus souvent assurée par des citoyens élus par leurs pairs et soumis à réélection. Le champ d'activité de ces collectivités recouvre tous les éléments des besoins individuels et sociaux de ses habitants. Selon le positionnement géoéconomique d'un territoire, ces préoccupations peuvent plutôt concerner le cadre de vie, l'aménagement du territoire, le tourisme, les conditions d'habitat, les services rendus en matière d'éducation, de santé, de culture, d'approvisionnement, de lutte contre les pollutions ou de mobilité, etc. Le domaine de la vie économique est également pris en charge, mais il demeure globalement 
marginal: aide à l'insertion professionnelle, attraction d'unités de production, promotion de l'emploi...

A la différence de la notion de «territoire », celle de «territoire économique » n'a pas de reconnaissance juridique (sauf quelques tentatives comme les « bassins d'emploi » ou les « pays » sans grandes portées opérationnelles). De fait, la vision qui est sous-jacente à cette notion, à savoir, un travail territorial sur l'économie locale, est peu développé. Comme il a été indiqué supra, il s'agit de mesurer les flux d'entrée et de sortie des personnes (éventuellement des biens et des mouvements financiers) du territoire administratif et d'en tirer des conclusions quant à la mise en œuvre de politiques volontaristes en matière d'attractivité.

\section{Quelques pistes possibles pour des territoires numériques cherchant à concurrencer les entreprises}

Il semble que la matrice primaire d'une stratégie territoriale conquérante ${ }^{12}$ soit une bonne compréhension de la notion de « territoire économique ».

- la première phase consiste à connaître les flux de mobilité des « déplacements domicile-travail » des habitants du territoire, ainsi que la provenance géographique des personnes qui viennent y travailler. L'Insee fournit, pour l'ensemble des communes (France métropolitaine et DOM) les effectifs correspondant aux croisements du lieu de résidence avec le lieu de travail ;

- dans une deuxième phase, il conviendrait d'établir une carte isochrone des déplacements possibles entre différents points du territoire et des zones extérieures, accessibles, aller et retour, au cours de la même journée ;

- le cœur de la démarche économique des territoires est de chercher à attirer des personnes habitant et travaillant en zone urbaine ; autrement dit, à attirer des postes de travail (contre la logique brick and mortar dominante);

- une politique volontariste d'équipements dédiés, directement ou non au télétravail, doit être le centre de la nouvelle politique économique territoriale; cette stratégie peut chercher à combiner les logiques top down et bottom up et inclure une importante dimension horizontale (voir Annexe) ;

- enfin, il convient d'asseoir l'ensemble de la démarche sur la politique locale de recherche générale de l'attractivité du territoire.

On peut néanmoins s'interroger sur le fait de savoir si la mise en œuvre d'un tel dispositif - même s'il est appliqué pleinement (de façon complète, et à tous les territoires composant la société nationale); harmonieusement (logique de système); efficacement (association de tous les acteurs locaux) - serait de nature à amener le territoire à devenir le moteur de la vie économique d'une nation.

La réponse risque probablement d'être négative, dans la mesure où la production - dont on sait depuis Jean-Baptiste Say [SAY 96] et Marx [MAR 80] qu'elle constitue ce moteur de la vie d'une société - reste cantonnée dans le monde des entreprises. La hiérarchie entreprise-territoire serait donc condamnée à demeurer figée, avec son cortège de blocages et de désespérances, tant que l'entreprise gardera le monopole de la production.

Sauf que, une nouvelle technologie - l'impression en trois dimensions et les lieux où elle prospère, les Fabrications Laboratories (Fab Labs) [MOR 16] - née aussi de la révolution numérique peut venir mettre en cause cet équilibre (déséquilibre) ancestral. Avec cette nouvelle manière de fabriquer des objets, le territoire peut accéder au statut (supérieur) de producteur.

\footnotetext{
${ }^{12}$ Dans cette matrice, on peut faire figurer le mouvement des Commons [COR 15] [DAR 14] [LER 15].
} 
Cette idée d'une concurrence possible des entreprises par les territoires peut laisser incrédule tout observateur, même de bonne foi. Le dossier ne semble cependant pas tout à fait vide ; on peut y trouver quelques pièces convaincantes :

- les très rapides progrès de l'impression $3 \mathrm{D}$, en variété de produits et en extensions géographiques $^{13}$;

- l'existence de millions d'utilisateurs-concepteurs dans le monde réunis dans des communautés locales ou sur internet ;

- s'y retrouvent de purs amateurs, des bricoleurs, des professionnels, des entreprises ;

- ceux-ci échangent, le plus souvent gratuitement, les données nécessaires à la production de tel ou tel objet ;

- les objets sont fabriqués pour des besoins concrets des intéressés ou pour leur seul plaisir;

- ils sont fabriqués à l'unité, peuvent être facilement modifiés ;

- leur coût de revient est, le plus souvent, infime ;

- ils ne génèrent que peu de déchets, etc.

Plus globalement ce nouveau type de production peut être analysé comme un attribut (fondamental) de la révolution numérique (à ce jour, encore peu documenté) : celui de l'abondance. On le sait, la consommation d'une information ne la détruit pas mais peut même l'enrichir; un objet fabriqué par une imprimante 3D peut être reproduit, modifié ou non, en une "longue traîne » [AND 07] quasi infinie.

Depuis toujours - si l'on excepte la période de l'économie de sous-production des chasseurscueilleurs ${ }^{14}$ [SAH 72] - l'homme et ses sociétés successives n'ont connu que la rareté et les problèmes de gestion qu'elle suscitait. Toute la pensée économique a travaillé sur les meilleures formes d'allocation des ressources, de partage des richesses produites. Aujourd'hui, ces champs avancent vers l'obsolescence, tout comme la forme dominante de l'entreprise parait désormais datée ${ }^{15}$.

\section{Conclusion}

Le dossier « Télétravail » n'a que peu avancé au cours de ces dernières décennies, alors qu'il reçoit un accueil favorable chez de nombreux salariés, que diverses mesures de soutien et d'incitation aient été prises par les pouvoirs publics, et que des expériences concrètes, menées dans quelques entreprises significatives, se soient toutes réalisées à la satisfaction des managers et des collaborateurs. Cette apathie presque totale témoigne de la puissance de la force de résistance du système, qui reste maître de l'acceptation ou du refus d'incorporer dans la production des innovations issues des recherches techniques et technologiques.

Ceci posé, la question du télétravail a fait apparaître, dans le débat public, l'importance, pour un grand nombre de salariés, de la conflictualité entre vie professionnelle et vie personnelle. On pourrait avancer que cette conflictualité est consubstantielle au principe même du salariat : contre la mise à

\footnotetext{
${ }^{13}$ Le Maire de mon village de résidence m'a demandé d'assurer la présidence d'une association chargée de sensibiliser les enfants des écoles primaire et maternelle à la technologie de l'impression 3D. Les premiers résultats sont très encourageants.

${ }^{14}$ Lors d'un colloque à la Cité des Sciences et de l'Industrie, organisé par le Réseau de recherches sur l'innovation (RRI) j'ai avancé l’idée de caractériser cette nouvelle économie territoriale, comme un « néo-communisme primitif».

${ }^{15}$ Peut-être une voie pour le dépassement de la fétichisation de la marchandise ? En tout cas, les interrogations sont de plus en plus nombreuses quant aux conséquences du développement de ce type d'économie «pro-am » et, en particulier, ses effets sur l'emploi salarié [STI 15].
} 
disposition de l'employeur de ses capacités productives, l'individu perçoit un certain montant de monnaie, qu'il est libre d'utiliser à sa guise (on sait que cette liberté est largement factice, dans la mesure où les usages du pactole détenu par le salarié sont quasi totalement prédéterminés). Cet enfermement est redoublé par l'obligation faite au salarié de se loger à proximité du lieu d'exercice de son travail, où sont concentrées les machines et autres équipements de production. Le schéma serait acceptable en matière de déplacements (réduits) domicile-travail, si trois tendances récentes n'étaient venues le mettre en cause :

- les restructurations industrielles qui ont fortement contribué à casser ce lien de proximité ;

- le développement du secteur tertiaire, et la croissance élevée du nombre d'immeubles de bureau, beaucoup plus sensibles à la mobilité géographique ;

- les évolutions des marchés immobiliers et l'extension conséquente des périphéries urbaines.

La solution visant à rapprocher ces deux temps de la vie d'un individu n'a malheureusement pas engendré de résultats très tangibles. L'expérience de plusieurs décennies de tentatives d'implanter le télétravail dans le système économique capitaliste montre son incompatibilité avec le principe de fonctionnement de ce système, à savoir le taylorisme et ses développements en termes d'hypertaylorisme. En cela, cette expérience révèle, en creux, l'une des failles majeures de la société industrielle : son incapacité à faire vivre un mode d'organisation du travail favorable aux travailleurs, un processus de destruction créatrice qui serait positif pour l'individu.

Aujourd'hui, apparaissent de nouvelles opportunités technologiques (impression 3D, FabLab) formant peut-être- une vague capable de causer des destructions plus significatives dans ce système. On peut faire l'hypothèse que la "révolution numérique », tant de fois annoncée mais toujours inerte face au pouvoir des critères de la gestion capitaliste, atteint cette fois-ci, une autre dimension, donne un sens plus concret à la notion de « révolution », car elle tend à modifier en profondeur, le contenu même de la production. Celle-ci peut désormais être réalisée en dehors des usines, dans n'importe quel local, que ce soit le domicile d'un particulier, un FabLab local, une université, etc. Elle est réalisée à la demande d'une personne ou d'un groupe, pour la fabrication de tel ou tel objet (schéma qui élimine la question des débouchés et le risque de surproduction).

Il est désormais possible d'imaginer une évolution de la question de la conciliation des deux temps principaux de l'existence humaine : le temps consacré à la vie professionnelle et celui dédié aux activités personnelles. Les espoirs placés dans le télétravail pour rapprocher et harmoniser ces deux temporalités n'ont pas abouti, laissant la question largement non résolue. Avec la révolution de la production personnelle, cette question peut se trouve dépassée par une autre, beaucoup plus riche de perspectives : celle de la fusion de ces deux temps en un seul.

Si cette hypothèse se vérifiait, on pourrait alors voir émerger une nouvelle "longue période », succédant à celle du monde industriel, où une force suffisamment puissante pourrait commencer à marginaliser le taylorisme ; elle transformerait, de façon dirimante, le statut du travail : celui-ci, passé de son modèle artisanal pour devenir celui du salariat, atteindrait un nouveau stade, qualifiable de «néo-artisanal, où se combineraient travail des amateurs et des professionnels, travail individuel et collaboratif, réalisé au sien de communautés locales ou réticulaires, fonctionnant sur la base de la confiance, et de la gratuité, aptes à gérer le nouveau monde de l'abondance...

A l'évidence, les questions de mobilité se situeraient dans un tout autre contexte, celui de la tendance à l'égalisation dynamique de la valeur des territoires ${ }^{16}$ marquant la fin des exodes ruraux et l'accélération des exodes urbains. Une des conditions pour que ce schéma, aujourd'hui techniquement accessible, se mette en place serait une mobilisation politique impliquant les élus locaux comme

\footnotetext{
${ }^{16}$ Un bon exemple de ce «monde nouveau » est celui de la ville de Barcelone, qui cherche à devenir autosuffisante en 2030.
} 
«entrepreneurs de l'économie territoriale», les habitants de ces territoires en formant l'élément moteur.

Reprenant - mais avec d'autres moyens - la logique ancienne du monde villageois et artisanal, l'objectif de réunir en un seul les temps professionnel et privé, pourrait constituer le programme de ces communautés.

\section{Bibliographie}

[AND 07] ANDERSON C., La longue traîne, quand vendre moins, c'est vendre plus, Flammarion, Paris, 2007.

[COR 15] CORIAT B., Le retour des communs, la crise de l'idéologie propriétaire, Les liens qui libèrent, Paris, 2015.

[DAL 17] DALIO R., Principles, 2017 (à paraître).

[DAR 14] Dardot P., LAVAl C., Commun, essai sur la révolution du XXI ${ }^{\mathrm{e}}$ siècle, La Découverte, Paris, 2014.

[DIE 57] DiEBOLD J., Automatisme (automation) vers l'usine numérique, Dunod, Paris, 1957.

[LAR 11] LARTAil M., Siben C., Bettinelli B., Perspectives de développement du télétravail dans la fonction publique, CGIET, Paris, 2011.

[LER 17] Fab labs, télétravail, un mouvement de convergence vers un emploi durable, in Boutillier Sophie (dir.) Entrepreneuriat et innovation, contextes et concept, P. I. E. Peter Lang, Bruxelles, Bern, Berlin, Frankfurt am Main, New York, Oxford, Wien, 2017, pp. 109-130.

[LER 16] Etude sur le télétravail en France : Volet France de Inquiry on Telework in Europe, Ministry of I nternal Affairs and Communications, Japan, 12 février 2016.

[LER 15] Vers l'émergence d'un artisanat collectif: les ouvertures offertes par les Fab Labs, du village à la communauté », in Boutillier Sophie, Fournier Claude, Perrin Cédric, Le temps des artisans, permanences et mutations, Marché et Organisations, L'Harmattan, Paris, 2015, pp. 173-200.

[LER 12] Communication : « Le télétravail comme inauguration d'une nouvelle séquence Technologie-Travail-Territoire, axe de construction collaborative de la proximité », $7^{\mathrm{e}}$ Journées de la Proximité « Créativité, Innovation et Proximité », HEC Montréal, 21-23 mai 2012.

[LER 09] Le travail collaboratif, une innovation générique, Marché \& Organisations, L’Harmattan, Paris, 2009,206 p.

[LER 04] Document de travail «Temps et subsomptions d'hier et d'aujourd'hui. La situation actuelle des conflits sociaux » LabRII-ULCO, 2004, 28 p.

[LOW 76] LOWE A., The Path of Economic Growth, Cambridge University Press, 1976.

[LUC 89] LUCAS Y., Le vol du savoir, techniciens de l'aéronautique et évolution des technologies, Presses universitaires de Lille, 1989.

[MAR 80] MARX K., Introduction de 1857, Manuscrits de 1857-1858 (Grundrisse), Editions sociales, Paris, 1980.

[MOR 16] MOREL L., LE RouX S., Fab Labs, l’usager-innovateur, ISTE Editions, London, 2016.

[PAC 94] PACIOLI L., Summa di arithmetica, proportioni et proportionalita, Venezia, 1494 (traduction de Haulotte \& Stevelinck, Vesoul, n. d.).

[SAH 72] SAHLINS M., Age de pierre, âge d'abondance, l'économie des sociétés primitives, Gallimard, Paris, 1972.

[SAY 96] SAY J.-B., Cours d'économie politique et autres essais, Flammarion, Paris, 1996.

[SOM 92] SOMBART W., Der Moderne Kapitalismus, 1916 (traductions partielles de : Lane \& Riemersma, Enterprise and secular change, readings in economic history, Allen \& Unwin, London, 1953, pp. 25-40 ; Nikitin, Cahiers d'histoire de la comptabilité, $n^{\circ} 2,1992$, pp. 29-35).

[STI 15] STIEGLER B., L’emploi est mort, vive le travail, Editions Mille et Une nuits, Paris, 2015.

[TAY 90] TAYLOR F. W., Organisation du travail et économie des entreprises, Editions d'Organisation, Paris, 1990.

[WIE 48] WIENER N., Cybernetics, John Wiley \& Sons, New York, 1948. 


\section{Type de préconisation}

\section{pour un réseau d'équipements dans une collectivité territoriale}

1. Un télécentre et un Fab Lab, nœud central du réseau, doté d'équipements et de compétences de qualité

2. Des télécentres-relais dans les principales zones de résidence du territoire

3. Des mini-télécentres locaux pouvant être installés dès qu'une demande apparaît (pour fournir à la population des solutions de proximité, évitant le plus possible des déplacements impliquant l'usage de l'automobile)

4. Des solutions d'assistance pour les travailleurs à domicile pouvant être reliés au réseau par des conventions-types

5. Une offre adaptée d'hébergement temporaire pour les personnes itinérantes (nomades, touristes, etc.)

6. Des relations avec les entreprises

7. Des partenariats avec les fournisseurs d'accès à internet

8. Un groupement des revendeurs d'équipements et dépanneurs

9. Des liens avec tous services publics et associatifs pour le développement d'applications spécialisées et échanges croisés

10. Des espaces de rencontres pour les professionnels et/ou les particuliers pour :
a. La promotion du territoire
b. La diffusion de l'offre touristique
c. L'accueil des visiteurs, en particulier la fourniture de listes de résidences disponibles à l'achat ou à la location, la liste des emplois non pourvus
d. La vente et la promotion de produits locaux
e. La sensibilisation des enfants, collégiens, lycéens
f. La mise en place de filières de formation
g. L'aide aux jeunes sans emploi
$h$. Le soutien aux personnes isolées ou en difficulté
i. Le développement des activités culturelles, sportives, etc. 